FEDSM2013-16349

\title{
MICROFLUIDIC DEVICE FOR SYNTHESIS OF CHITOSAN NANOPARTICLES
}

\author{
Barbaros Cetin* \\ Microfluidics \& Lab-on-a-chip Research Group \\ Mechanical Engineering Department \\ Ihsan Dogramaci Bilkent University \\ Ankara 06800 TURKEY \\ Email: barbaros.cetin@bilkent.edu.tr
}

\author{
Mehmet D. Asik \\ Nanotechnology and Nanomedicine Division \\ Hacettepe University \\ Ankara 06800 TURKEY \\ Email: mehmetdoganasik@gmail.com
}

\author{
Serdar Taze \\ Microfluidics \& Lab-on-a-chip Research Group \\ Mechanical Engineering Department \\ Ihsan Dogramaci Bilkent University \\ Ankara 06800 TURKEY \\ Email: serdar.taze@bilkent.edu.tr

\section{S. Ali Tuncel} \\ Nanotechnology and Nanomedicine Division \\ Hacettepe University \\ Ankara 06800 TURKEY \\ Email: atuncel@hacettepe.edu.tr
}

\begin{abstract}
Chitosan nanoparticles have a biodegradable, biocompatible, non-toxic structure, and commonly used for drug delivery systems. In this paper, simulation of a microfluidic device for the synthesis of chitosan nanoparticle is presented. The flow filed together with the concentration field within the microchannel network is simulated using COMSOL Multiphysics $\AA$ simulation environment. Different microchannel geometries are analyzed, and the mixing performance of these configurations are compared. As a result, a $3 D$ design for a microfluidics platform which includes four channel each of which performs the synthesis in parallel is proposed. Future research directions regarding the fabrication of the microfluidic device and experimentation phase are addressed and discussed.
\end{abstract}

\section{NOMENCLATURE}

$\mathrm{A}_{o}$ coefficient used in Eq. (3)

$\mathrm{A}_{1}$ coefficient used in Eq. (3)

$\mathrm{A}_{2}$ coefficient used in Eq. (3)

${ }^{*}$ Address all correspondence to this author.

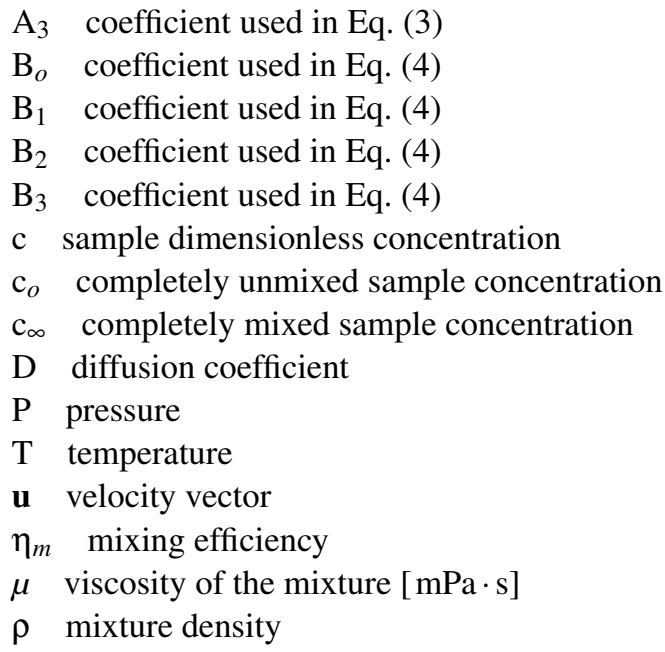

\section{INTRODUCTION}

Use of biopolymers dramatically increased in medicine, pharmacology and industry in last decade. The knowledge in recent literature suggest that the use of chitosans as safe bio- 
materials for various applications in biomedical sciences and in pharmaceutical sciences [1]. Chitosan is a hydrophilic macromolecule can form biodegradable nanoparticles for site specific delivery of vaccines, genes, drugs and other biomolecules in the body [2]. Moreover; it is used as a topical dressing in wound management due to its hemostatic, stimulation of healing, antimicrobial, nontoxic, biocompatible and biodegradable properties [3]. Also, chitosan has been considered as a promising candidate for bone tissue engineering with the properties of minimal foreign body reactions, the ability to be molded into various geometries and forms such as porous structures, suitable for cell ingrowth and osteoconduction [4].

The conventional methods for nanoparticle formation of chitosan include emulsion droplet coalescence, emulsion solvent diffusion, reverse micellar method, ionic gelation, polyelectrolyte complexation and desolvation [5]. These techniques are batchwise systems, mostly has the principle of dropwise addition of the cross-linker molecules to the chitosan solution or chitosan solution to the cross-linker solution. However, conventional techniques sometimes suffer from aggregation of nanoparticles and requires a qualified personnel throughout the process due to the lack of automation. Moreover, reproducibility of the process may be also problematic. One alternative to overcome these issues is to synthesize the chitosan nanoparticles within the microchannels in a continuous flow. In this case, the flow hence the process can be performed in a more controlled manner, the aggregation can be reduced due to the continuous flow nature of the process, and the process can be run autonomously once the required solutions are loaded into the microfluidic channels.

The main challenge of synthesis is the mixing of the nanoparticles with TPP solution. Due to low Reynolds number nature (i.e. laminar flow) of the microchannel flows, mixing of species becomes a challenge due to the absence of turbulence. The proper mixing in a microchannel can be achieved with various channel design (passive mixers) or introducing additional mixers such as ultrasonic mixer, electrokinetic forcing and alternate injection mixer (active mixers) [6]. Although, the use of mixer has positive effect on mixing efficiency, mixers also bring some difficulties such as additional cost and effort to tune the mixer on the microfluidic device. The easy and simple way of an efficient mixing is to utilize special design of the microfluidic network. Different designs have been proposed in the literature such as hydrodynamic focusing channels [6], zigzag microchannel [7], squarewave, three-dimensional serpentine and staggered herringbone mixer [8].

In this study, the numerical simulation of a continuous flow microchannel flow for synthesis of chitosan nanoparticles which requires mixing of two solutions in a prescribed ratio is presented. Although the mixing of mixtures within microchannels have been studied extensively, mixing study regarding the synthesis of chitosan nanoparticles has not been studied. The microfluidic structures used in the simulations have three inlet

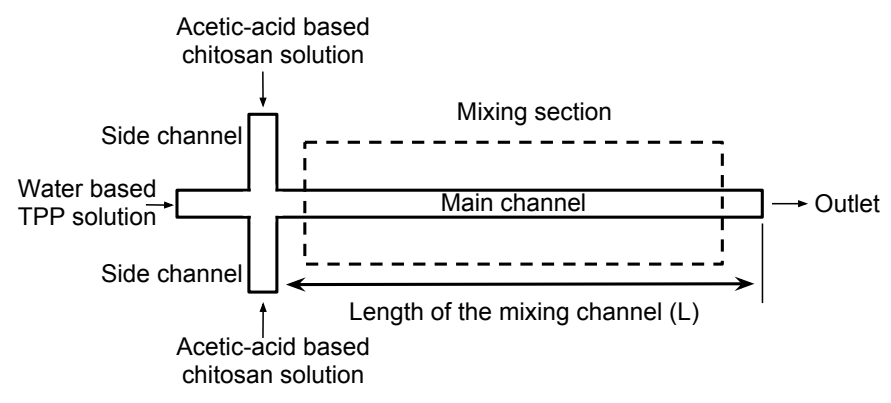

Figure 1. THE SCHEMATIC DRAWING OF THE MICROCHANNEL NETWORK

reservoirs, single exit reservoir. Micro obstacle structures as proposed in [9] are introduced within the microchannels to increase the mixing efficiency. In the simulations, only 2D models are considered and the mixing efficiency of several configurations are compared. As a result, a 3D design for a microfluidics platform which includes four channel each of which performs the synthesis in parallel is proposed and a fabrication procedure based on micromachining is introduced. The experimentation procedure is also discussed to address future research direction.

\section{ANALYSIS}

A microfluidics channel network with 3 inlet reservoirs and 1 exit reservoir is proposed for the synthesis of chitosan nanoparticles as shown in Fig. 1. Following the well-known ionic gelation method, the synthesis requires dropwise mixing of acetic acid based chitosan polymer solution with the water based TPP solution with a certain ratio. In a typical ionic gelation chitosan nanoparticle synthesis, mixing ratio of TPP:chitosan ratio varies in between 1:2 to 1:5. In this study, ratio of $1: 3$ is chosen. In a typical protocol, $1 \% 100 \mathrm{~mL}$ acetic acid solution is prepared and $0.5 \mathrm{gr}$ chitosan (low molecular weight, degree of deacetylation $80 \%$ ) is dissolved in this solution. Separately, $0.5 \%$ (weight/volume) TPP solution is prepared. Then, $2 \mathrm{~mL}$ of TPP solution is added to $6 \mathrm{~mL}$ chitosan solution in 10 mins time in a dropwise manner. To ensure the same order of magnitude throughput, the volumetric flow rate of the acetic acid and water solutions are taken as $0.15 \mathrm{~mL} / \mathrm{min}$ and $0.05 \mathrm{~mL} / \mathrm{min}$. the width and height of the main channel and the side channels are taken as $1000 \mu \mathrm{m}$, and $500 \mu \mathrm{m}$ respectively. To evaluate the mixing efficiency of the microchannel network, flow and concentration fields needs to be determined. In this model, following assumptions are used:

(1) the working fluids are incompressible, Newtonian liquids,

(2) the gravitational effects and buoyancy effects are negligible,

(3) both species have identical diffusion coefficients,

(4) no chemical interaction between the chitosan particles and 
the channel wall and no chemical reactions take place.

(5) the mixing of two liquids ensures the mixing of chitosan nanoparticles with TPP.

The flow field is governed by Navier-Stokes equation,

$$
\rho_{m} \mathbf{u} \cdot \nabla \mathbf{u}=-\nabla P+\mu \nabla^{2} \mathbf{u}
$$

subjected to the no-slip boundary conditions at the channel walls, specified flow rates at the reservoirs and zero pressure boundary condition at the exit. The concentration field is governed by convection-diffusion equation,

$$
\mathbf{u} \cdot \nabla c=D \nabla^{2} c
$$

subjected to insulated boundary at the channel walls and the specified concentration at the reservoirs. In here, $c$ represents the dimensionless mole fraction of the acetic acid. Therefore, $c$ is assigned as unity at the inlets of acetic acid, and assigned as zero for the inlets of water. At the exit, convective flux boundary condition is assigned.

In these equations, mixture density and mixture viscosity need to be described in terms of the concentration. The mixture density can be determined by using the following relation [10]:

$$
\begin{aligned}
& \rho=A_{o}+A_{1} T+A_{2} T^{2}+A_{3} T^{3} \\
& A_{o}=534.613+1950.54 c-1054.32 c^{2}+174.019 c^{3} \\
& A_{1}=4.1946-10.5253 c+3.15922 c^{2} \\
& A_{2}=-0.0113495+0.0212374 c-0.0036607 c^{2} \\
& A_{3}=8.43584 \times 10^{-6}-1.47636 \times 10^{-5} c
\end{aligned}
$$

To determine the mixture viscosity, following relation which relates the water-acetic acid mixture as a function of mole fraction of acetic acid is used [11]:

$$
\begin{aligned}
& \ln \mu=B_{o}+B_{1} / T+B_{2} \ln T+B_{3} P \\
& B_{o}=-9.84679+5.93224 c-0.0724913 c^{2}+1.59018 c^{3} \\
& B_{1}=2361.48+202.364 c-1594.8 c^{2} \\
& B_{2}=0.00609668-0.00856923 c \\
& B_{3}=0.014739
\end{aligned}
$$

The lowest temperature was given in [11] was $313 \mathrm{~K}$, so the same temperature is used in this study. The pressure value is taken as $100 \mathrm{kPa}$, since the typical pressure drops in liquid microfluidic applications are in the order of $100 \mathrm{~Pa}$ [9], the variation of viscosity with pressure is neglected.

In order to quantify the mixing performance, the mixing efficiency at the exit of the mixing section is determined. Mixing efficiency can be determined as [12],

$$
\eta_{m}=1-\frac{\int_{A}\left|c-c_{\infty}\right| d A}{\int_{A}\left|c_{o}-c_{\infty}\right| d A}
$$

where $c$ is the sample concentration, and $c_{o}$ and $c_{\infty}(=0.75)$ are the sample concentrations in the completely unmixed and completely mixed conditions, respectively. Therefore, $\eta_{m}=0$ indicates a completely unmixed state, and $\eta_{m}=1.0$ indicates complete mixing.

\section{NUMERICAL MODELING}

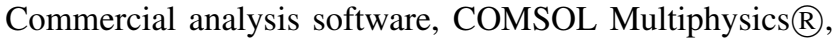
which is based on the finite element method, was used to determine the flow and concentration fields. Since the density and the viscosity of the fluid depend on the concentration of the acetic acid, and the velocities in $x$ - and $y$-directions are required to determine the convective terms in the convection-diffusion equation, the flow field and concentration field are coupled. Because the problem is nonlinear, stationary and nonlinear choices were used as solver parameter. Since the equations are coupled and nonlinear, it was observed that the convergence of the solution sensitive to initial guess. Therefore, to obtain the converged solution, the equations are solved in sequence by updating the initial guess from the previous run. First, the Convection-Diffusion module is solved based on the initial zero velocities (i.e. purely diffusion equation). After that the Incompressible Navier-Stokes module together with the results that are obtained from the diffusion equation is computed. Then, the convection-diffusion equation resolved with the obtained velocity field. One more iteration was performed for both flow field and concentration field to obtain the converged solution. The simulations were performed on a HP Z400 Workstation (Intel Xeon W3550, Quad

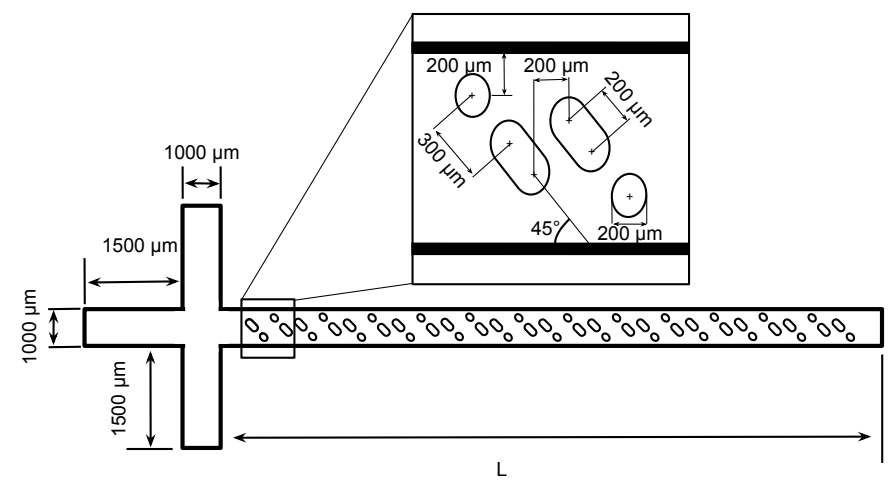

Figure 2. DRAWING OF THE MICROCHANNEL NETWORK WITH OBSTACLES 
Table 1. INPUT PARAMETERS FOR THE SIMULATIONS

\begin{tabular}{ll}
\hline Density of water & $\rho_{w}=1000 \mathrm{~kg} / \mathrm{m}^{3}$ \\
Density of acetic acid & $\rho_{a}=1050 \mathrm{~kg} / \mathrm{m}^{3}$ \\
Binary diffusion coefficient & $D=10^{-9} \mathrm{~m}^{2} / \mathrm{s}$ \\
L & $1,2,3,4,5 \mathrm{~cm}$ \\
\hline
\end{tabular}

core, $3.06 \mathrm{GHz}$, 16GB RAM). Mesh independence was checked, and during the mesh generation step maximum mesh size of $60 \mu \mathrm{m}$ with a $\mathrm{n}$ element growth rate of 1.1 were specified. The mixing efficiency of each configuration was determined based on the concentration profile at the channel exit. To determine the case for completely unmixed state $\left(c_{o}\right)$, the simulation is performed with very small diffusion coefficient.

\section{RESULTS AND DISCUSSION}

Two microchannel structures, one of which is a straight channel (see Fig. 1) and one of which is a channel with obstacles (see Fig. 2) are simulated. The parameters used in the simulation are tabulated in Tab. 1. Binary diffusion coefficient is

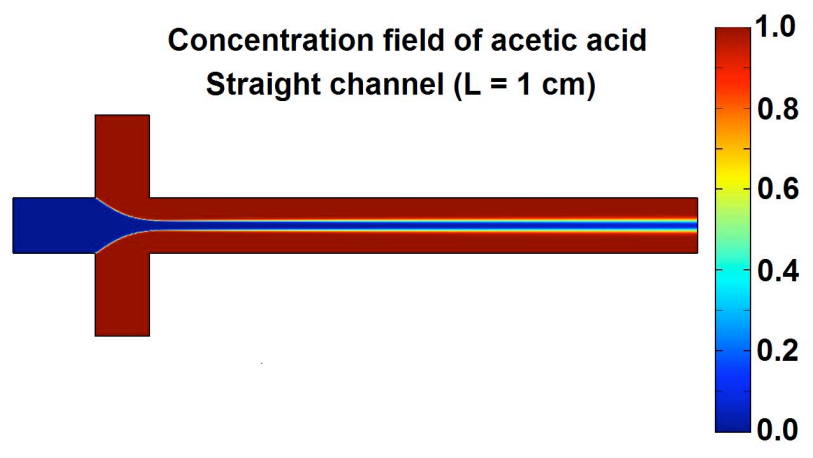

(a)

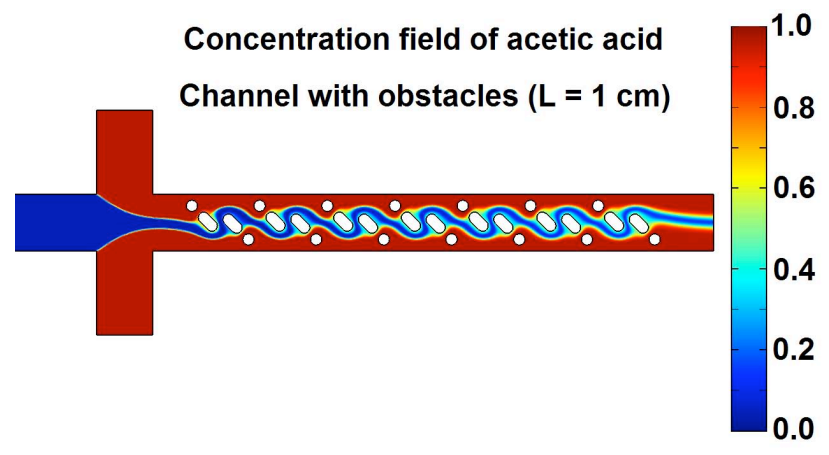

(b)

Figure 3. CONCENTRATION FIELD taken as $1 \times 10^{-9} \mathrm{~m}^{2} / \mathrm{s}$ which a typical value for aqueous solutions [9]. Fig. 3 shows the concentration field for straight channel and channel with obstacles with a length of $1 \mathrm{~cm}$. As seen from the figure, the channel with obstacles has a better mixing than that of a straight channel for a given length. Another important observation is that, for straight channel concentration profile symmetric around the center; however, for the channels with obstacles the symmetry is broken for some cases which due to the presence of the asymmetric obstacles within the microchannel.

For a better mixing efficiency, length of the mixing channel $(L)$ is an important parameter. Therefore, microchannel geometries with different mixing channel length $(L=1,2,3,4,5 \mathrm{~cm})$ are simulated. The concentration profile of acetic acid at the exit of the microchannel are given in Fig. 4. As the obstacles are introduced, and the concentration profile improves from completely

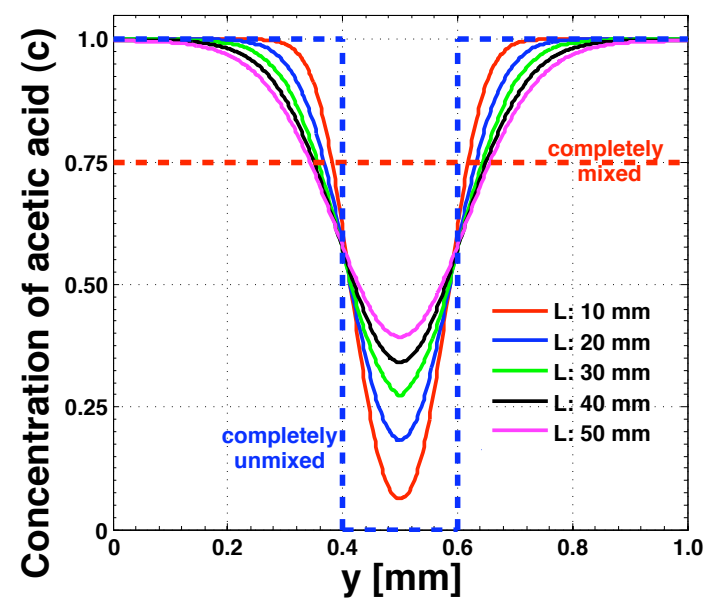

(a)

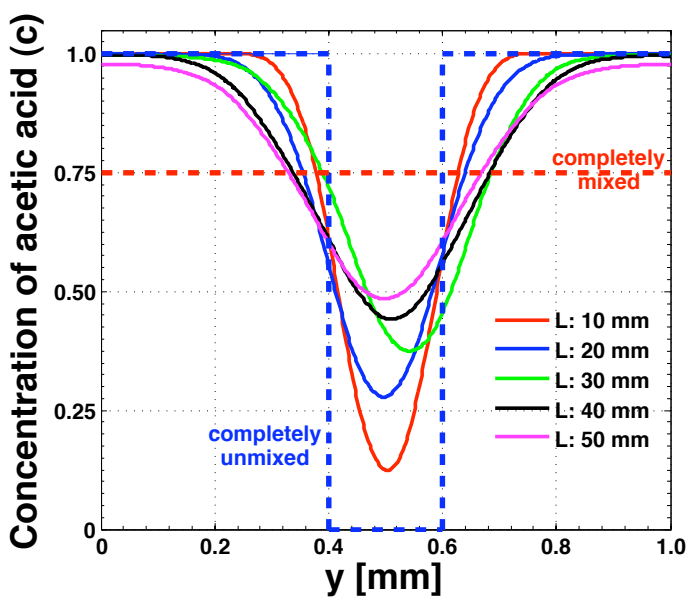

(b)

Figure 4. CONCENTRATION OF ACETIC ACID AT THE EXIT OF THE CHANNEL 


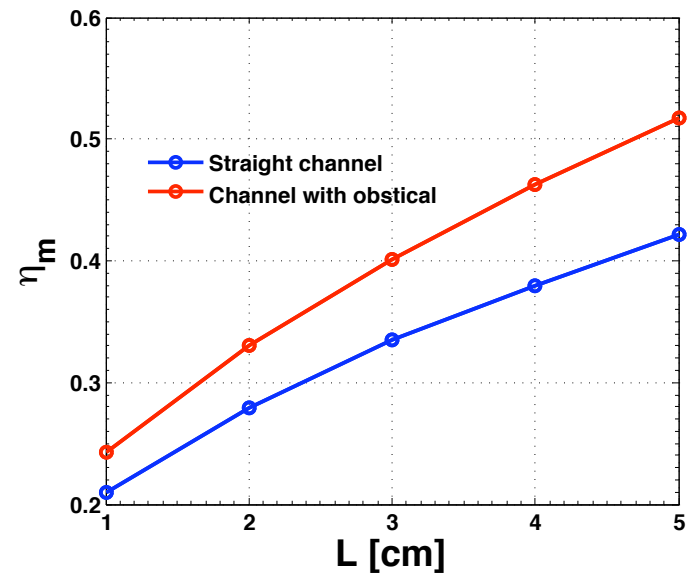

Figure 5. MIXING EFFICIENCY FOR DIFFERENT CHANNEL CONFIGURATION

unmixed condition to completely mixed condition. To quantify the performance of mixing, the corresponding mixing efficiencies are also illustrated in Fig. 5 as a function of length of the mixing channel. As seen from the figures, the mixing efficiency improves as the obstacles are introduced and as the length of the mixing channel increases.

Following these results, an S-shaped microchannel with obstacles is considered. S-shape channel geometry enhances mixing due the presence of the inertia in radial direction, and is more compact compared to the previous configurations. The technical drawing of the S-shaped microchannel is shown in Fig. 6. The same configuration is also proposed with a longer mixing channel geometry. The concentration fields for two different S-shaped microchannels are illustrated in Fig. 7. As seen from the figure, long S-shape channel has a superior mixing performance com-

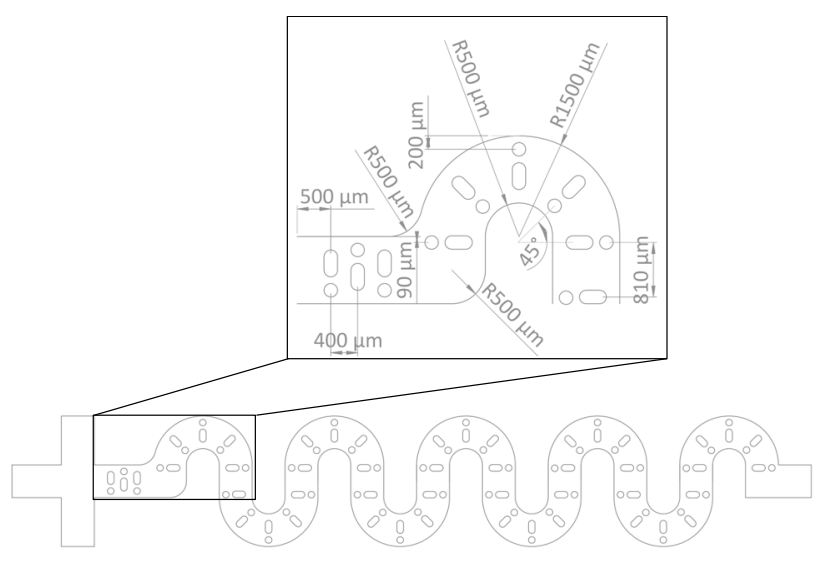

Figure 6. DRAWING OF THE S-SHAPED MICROCHANNEL
Table 2. $\eta_{m}$ FOR DIFFERENT CHANNEL CONFIGURATIONS

\begin{tabular}{lcccccc}
\hline \multirow{2}{*}{ Channel } & \multicolumn{6}{c}{$\mathrm{L}[\mathrm{cm}]$} \\
\cline { 2 - 7 } Configuration & 1.0 & 2.0 & 3.0 & 4.0 & 5.0 & 7.0 \\
\hline Straight & 0.210 & 0.299 & 0.335 & 0.380 & 0.421 & - \\
W/ obstacles & 0.242 & 0.331 & 0.401 & 0.463 & 0.517 & - \\
S-shape & - & - & - & 0.492 & - & 0.685 \\
\hline
\end{tabular}

pared to other configurations. Mixing efficiency values of all the configurations considered in this study are tabulated in Tab. 2. As seen form the table, long S-shape channel with a relatively compact design has a mixing efficiency of approximately $70 \%$ which is an acceptable limit for most of the mixing applications.

Since S-shape channel has an enhanced performance, a microfluidics platform which consists of four S-shape microchannel configuration in parallel with 4 inlet ports for acetic acid based chitosan solution, single inlet port for water based TPP solution and two outlet ports for the solution with sythentized nanoparticles is proposed. The CAD drawing of the proposed system can be seen in Fig. 8.

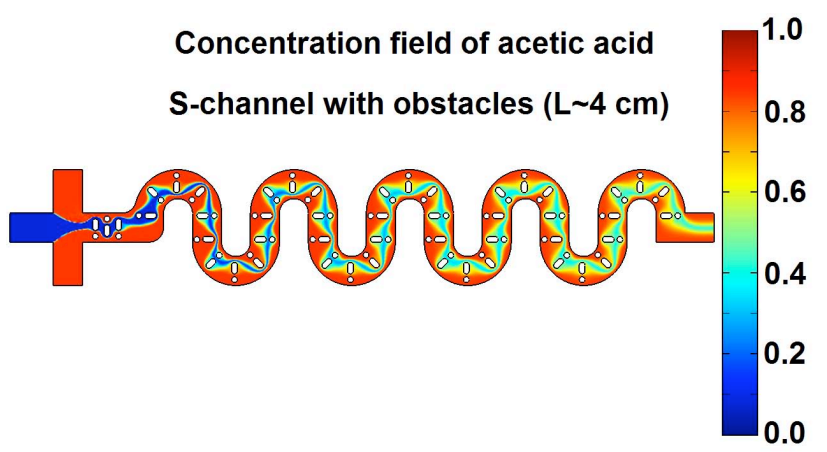

(a)

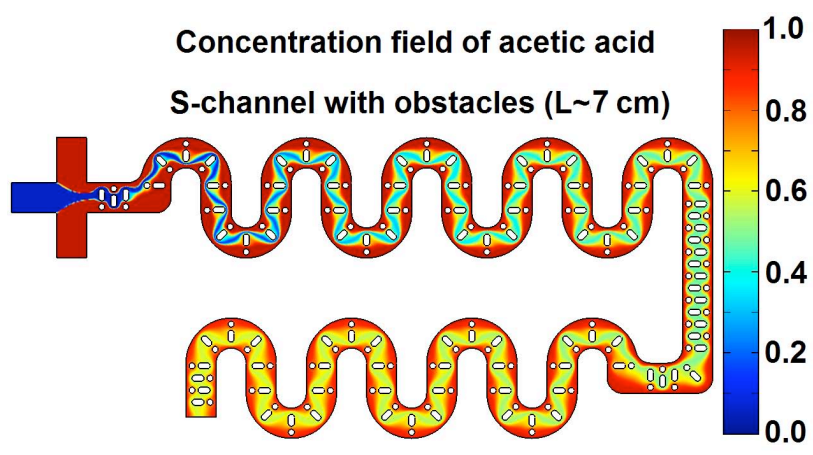

(b)

Figure 7. CONCENTRATION FIELD FOR S-CHANNELS 


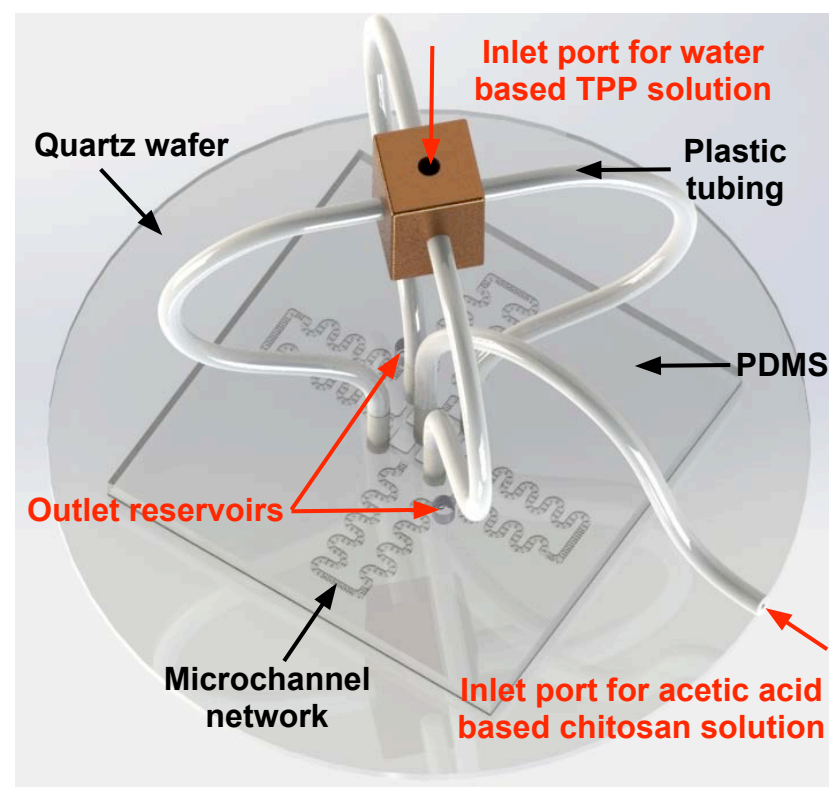

Figure 8. CAD DRAWING OF THE MICROFLUIDC DEVICE

\section{SUMMARY AND FUTURE WORKS}

In this study, the mixing of acetic acid based chitosan solution and water based TPP solution is simulated by using COM-

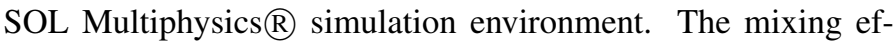
ficiency of different channel configurations and geometries are analyzed. For this study, only passive mixers are considered and proposed. The proposed microfluidic system will have the potential to be used for the synthesis of chitosan nanoparticles which are the key ingredients for many drug delivery systems, and will have advantages over the conventional batchwise system considering the continuous flow nature which will reduce the aggregation of the synthesized nanoparticles. In the experimentation phase, the flow of the solutions will be driven by syringe pumps with high accuracy, and controlled by computer which will enhance the reproducibility and the automation of the process. The products collected at the outlet of the channels will be characterized with zeta-sizer and scanning electron microscope to verify the nanoparticle synthesis and to determine the size distribution and zeta potential of the nanoparticles. The quality of the synthesis process performed within microfluidic device is compared with that of the conventional, bench-top equipments. Although only passive mixers are considered in this study, in the experimentation phase, active mixers like pulsating flow at the channel inlet [12] or ultrasonic mixer [6] may also be considered for even better performance.

Since the dimensions of the microchannel are in millimeter range and the size of the proposed obstacle structures are around $200 \mu \mathrm{m}$, for the fabrications of the microchannel network, the micromachining facility of the Bilkent University Micro System
Design and Manufacturing Center will be used. Either an aluminum mold will be micro-machined and the microchannel network will be fabricated out of PDMS or the channel structure will directly be micro-machined on a plexiglass substrate. Depending on the limitations on the fabrication, full 3D geometry will be determined and 3D simulations will be performed for the proposed geometry before the experimentation phase.

\section{ACKNOWLEDGMENT}

The authors gratefully acknowledge financial support for Mr. Serdar Taze by Bilkent University Mechanical Engineering Department.

\section{REFERENCES}

[1] Muzzarelli, R. A. A., 2011. "Biomedical exploitation of chitin and chitosan via mechano-chemical disassembly, electrospinning, dissolution in imidazolium ionic liquids, and supercritical drying". Marine Drugs, 9, pp. 15101533.

[2] Mahapatro, A., and Singh, D. K., 2011. "Biodegradable nanoparticles are excellent vehicle for site directed in-vivo delivery of drugs and vaccines". J. Nanobiotechnology, 9(55), pp. 1-11.

[3] Dai, T., Tanaka, M., Huang, Y.-Y., and Hamblin, M. R., 2011. "Chitosan preparations for wounds and burns: antimicrobial and wound-healing effects". Expert Rev Anti Infect Ther., 9(7), pp. 857-879.

[4] Venkatesan, J., and Kim, S.-K., 2010. "Chitosan composites for bone tissue engineering-An overview". Marine Drugs, 8, pp. 2252-2266.

[5] Grenha, A., 2012. "Chitosan nanoparticles: a survey of preparation methods". Journal of Drug Targeting, 20(4), pp. 291-300.

[6] Suh, Y. K., and Kang, S., 2010. "A review on mixing in microfluidics". Micromachines, 1(3), pp. 82-111.

[7] Mengeaud, V., Josserand, J., and Girault, H. H., 2002. "Mixing processes in a zigzag microchannel: Finite element simulations and optical study". Analytical Chemistry(16), pp. 4279-4286.

[8] Liu, Y., Kim, B., and Sung, H., 2004. "Two-fluid mixing in a microchannel". International Journal of Heat and Fluid Flow, 25, pp. 986-995.

[9] 1A. A. S. Bhagat, and Papautsky, I., 2008. "Enhancing particle dispersion in a passive planar micromixer using rectangular obstacles". J. Micromech. Microeng., 18, pp. 1-9.

[10] Sun, T., Ly, D., and Teja, A. S., 1995. "Densities of acetic acid + water mixtures at high temperatures and concentrations". Ind. Eng. Chem. Res., 34, pp. 1327-1331.

[11] Qiao, Y., Di, Z. G., Ma, Y. G., Ma, P. S., and Xia, S. Q., 2010. "Viscosities of pure water, acetic acid + water, and 
p-xylene + acetic acid + water at different temperature and pressure". Chinese Journal of Chemical Engineering, 18(3), pp. 446-454.

[12] Chen, C.-K., and Cho, C.-C., 2008. "A combined active/passive scheme for enhancing the mixing efficiency of microfluidic devices". Chemical Engineering Science, 63, pp. 3081-3087. 